\title{
GREP: a Group REkeying Protocol Based on Member Join History
}

\author{
Marco Tiloca \\ SICS Swedish ICT AB, Security Lab \\ Isafjordsgatan 22, Kista (Sweden) \\ Email:marco@sics.se
}

\author{
Gianluca Dini \\ Dipartimento di Ingegneria dell'Informazione \\ University of Pisa, Largo Lazzarino 1, Pisa (Italy) \\ Email: gianluca.dini@ing.unipi.it
}

\begin{abstract}
This paper presents GREP, a highly scalable and efficient group rekeying protocol with the following merits. First, it rekeys the group with only two messages, introducing an overhead which is small, constant, and independent of the group size. Second, GREP considers collusion as a first-class attack. Third, GREP efficiently recovers the group from a collusion attack without recourse to a total member reinitialization. The recovery cost smoothly grows with the group size, and gradually increases with the attack severity. GREP achieves these results by organizing nodes into logical subgroups and exploiting the history of node joining events. This allows GREP to establish a total ordering among subgroups and among nodes in each subgroup, so making collusion recovery highly scalable and efficient. We evaluate performance from several standpoints, and show that GREP is deployable in large-scale networks of customary, even resource constrained, platforms.
\end{abstract}

\section{Introduction}

Group communication is a powerful and efficient paradigm that can be used in a range of application scenarios, from wireless sensor networks (WSNs) to large scale distribution of contents. According to this model, a node becomes member of the group by explicitly joining it. After that, it may send/receive broadcast messages to/from other group members. Later on, the node may voluntarily leave the group or be forced to, if compromised or suspected so.

It is generally required that only group members can access group communication. To this end, group members secretly share a cryptographic group key to securely exchange messages in the group. When a node joins the group, it must be prevented from deciphering previous messages even if it has recorded them (backward security). When it leaves the group, or is forced to leave, the node must be prevented from further accessing group communication (forward security). Backward and forward security are generally guaranteed by rekeying. That is, when a node joins or leaves the group, the group key is revoked and a new one is distributed. In

\footnotetext{
. This work was carried out during the tenure of an ERCIM "Alain Bensoussan" Fellowship Programme. The research leading to these results has received funding from the European Union Seventh Framework Programme (FP7/2007-2013) under grant agreement $\mathrm{n}^{\circ} 246016$.
}

large dynamic groups where joining and leaving events are frequent, rekeying must be efficient and highly scalable.

Besides, a collusion attack occurs when multiple compromised group members share their security material, in order to regain access to the group key. No group rekeying scheme is exempt from collusion attacks, and different schemes display different levels of resilience. However, only a few of them consider collusion as a first-class attack, and provide countermeasures to recover from successful instances of this kind of attack. In many schemes, recovering from collusion requires a total member reinitialization, i.e. all non compromised group members have to be separately reinitialized, in a one to one fashion. It follows that the recovery overhead grows linearly with the group size, with negative impact on the overall system performance and scalability.

In this paper, we take these challenges and present GREP, a novel rekeying scheme for large-scale dynamic groups that levers on logical subgrouping and join history. Group members are partitioned into non overlapping logical subgroups that become the units of rekeying and collusion recovery. Unlike other schemes, subgroups only support efficient group key management, have no application meaning and are transparent to the application layer. Also, GREP exploits the history of joining events to establish a total ordering among subgroups and among nodes in each subgroup, in order to efficiently recover from collusion attacks.

GREP displays the following benefits. First, it is secure, as it fulfills the backward and forward security requirements. Second, it is highly scalable, as it requires a number of rekeying messages which is small, constant, and independent of the group size, i.e. $\mathcal{O}(1)$. Third, GREP has a $\mathcal{O}(\sqrt{n})$ storage and computing overhead, where $n$ is the group size. This makes the scheme deployable on a large spectrum of platforms, including resource scarce sensor nodes. Fourth, GREP considers collusion as a firstclass attack. Fifth, GREP efficiently recovers from collusion attacks, displaying a communication overhead that gradually increases with the attack severity, and grows as $\mathcal{O}(\sqrt{n})$ only in the unlikely worst case. This is possible by exploiting the history of joining events in the group. To the best of our knowledge, GREP is the first group rekeying protocol that exploits the join history to achieve highly efficient collusion recovery. 
The paper is organized as follows. Section 2 discusses related works. Section 3 describes the system architecture. We present the GREP protocol in Section 4, provide a security analysis in Section 5, and evaluate performance in Section 6. Section 7 draws our conclusive remarks.

\section{Related work}

Like other rekeying schemes suitable for large groups [2] [3] [6] [7] [10], GREP takes a centralized approach to group key management and relies on logically organized $a d$ ministrative keys to provide scalable and efficient rekeying.

LKH organizes administrative keys in a hierarchical logical tree [3], where the root contains the group key, the leaves contain group members' individual keys, and the internal tree nodes contain additional administrative keys. Given a group size $n$ and a balanced key tree with arity $a$, the leave communication overhead and the storage overhead at the node side grow as $\mathcal{O}\left(\log _{a} n\right)$. In case of collusion attack, LKH incurs the risk of a total member reinitialization when at least $\left\lceil\frac{n}{a}\right\rceil$ nodes are captured before they are detected.

Other schemes deriving from LKH have been proposed, but none of them achieves better performance [2] [6] [10]. Key Graphs is a generalization of LKH, where performance depends on the specific graph topology [2]. LARK is based on Key Graph, and relies on logically organizing administrative keys as a tool for application design [6]. That is, the key graph topology reflects cooperation within the group, and is considered to provide efficient rekeying. KTR adopts an approach similar to the one of LARK, and generalizes LKH to manage multiple subscriptions in content distribution applications and wireless broadcast services [10].

HISS [7] and GREP display similarities, as they both rely on logical subgrouping to support efficient and scalable rekeying. They achieve the same efficiency as to computing and storage overhead, i.e. $\mathcal{O}(\sqrt{n})$, and rekey the group with a number of messages which is reduced, constant, and independent of the group size. However, unlike HISS, GREP introduces the notion of member join history, and exploits it to recover from collusion attack in a much more efficient and scalable way. That is, the HISS recovery overhead always grows as $\mathcal{O}(n)$. Instead, the GREP recovery overhead gradually increases with the collusion attack severity, and grows as $\mathcal{O}(\sqrt{n})$ only in the unlikely worst case condition. Also, the chance of a total member reinitialization requires at least $2 \cdot\lceil\sqrt{n}\rceil$ colluding nodes, but is practically an unlikely event, so making GREP extremely efficient against collusion attacks even when several nodes collude.

\section{System architecture}

We consider a set $G$ of nodes that communicate according to the group communication paradigm. A node becomes member of $G$ by explicitly joining the group. Then, it may send/receive broadcast messages to/from other group members. If a member leaves the group, or is forced to, it cannot send/receive messages to/from the group anymore.
Group members secretly share a cryptographic group key that they use to encrypt/decrypt messages within the group. We denote by $K_{G}$ the group key associated to the group $G$. In general, it is required that the backward and forward security requirements are guaranteed [11]. In order to fulfill them, when a new node joins the group or a current member leaves it, the current group key is revoked and a new one is distributed. Hereafter, we refer to this operation as rekeying.

The group $G$ is managed by a Group Controller (GC), which is composed of three services: i) a Group Membership Service (GMS); ii) a Key Management Service (KMS); and iii) an Intrusion Detection Service (IDS). In short, the GMS maintains the group membership by keeping track of nodes that join and leave. The IDS component monitors network activities to detect possible compromised nodes. Since there is no sure and efficient way to readily detect a single node capture [12], the IDS may report multiple compromised nodes at the same time. Upon detecting a set of compromised nodes $G_{c}$, the IDS notifies the GMS in order to have them evicted from the group. Further details about the IDS and the monitoring process are beyond the scope of this paper, and we refer the reader to, e.g., [5] [13]. Upon being notified of any membership change, the GMS activates the rekeying process. The KMS is responsible for performing such a task.

The GC is typically implemented according to a centralized approach, as a resourceful computing node which is generally more powerful than group nodes. In particular, the GC is considered trustworthy and properly designed, implemented and managed to be reliable and secure, hence practically infeasible to compromise. Although server security and reliability are still an open research issue, the literature provides well established techniques to keep servers secure [4] [9]. As an alternative, the GC can be practically implemented according to a distributed architecture, which is beneficial in terms of robustness and availability, and avoids a single GC instance from being a single point of failure. However, this requires that the different GC replicas are kept synchronized with one another, especially as to the current group membership and established key material. In this paper, we consider a centralized GC, and detail the Key Manager (KM) component implementing the KMS. Further details about practical architectural design choices for the GC are out of the scope of this work.

\section{The rekeying protocol}

The group $G$ is partitioned into a set $\mathcal{S}$ of non empty subgroups, such that each group member is exactly in one of these subgroups. Subgroups have no meaning to applications and are never merged nor split. Each member of $G$ is assigned to a given subgroup $S$ upon joining the group, and is never moved to a different subgroup. We denote two nodes in the same subgroup as cognates.

GREP totally orders the members of each subgroup $S$ according to their joining time, to reflect the node join history of $S$. In particular, upon joining a subgroup $S$, a node $u$ is associated with a numeric node ID, nid $d_{u}$, which 
is unique within the subgroup, and reflects its members' total order. That is, given two nodes $u$ and $v$ in $S, n i d_{u}<n i d_{v}$ if and only if node $u$ has joined $S$ before node $v$. We refer to the nodes that have joined subgroup $S$ before or after node $u$ as elder cognates and junior cognates of $u$, respectively.

GREP also totally orders subgroups according to their addition time to group $G$, to reflect the subgroup addition history of $G$. In particular, upon its addition to the group $G$, a subgroup $S$ is associated with a numeric subgroup $\mathrm{ID}, \operatorname{sid}_{S}$, which is unique within the group $G$, and reflects subgroups' total order. That is, given two subgroups $S$ and $S^{\prime}, \operatorname{sid}_{S}<\operatorname{sid}_{S^{\prime}}$ if and only if subgroup $S$ has been added to $G$ before subgroup $S^{\prime}$. We refer to the subgroups that have been added to the group $G$ before or after subgroup $S$ as elder kindreds and junior kindreds of $S$, respectively.

Each node and subgroup is associated with secret quantities called tokens. That is, node tokens and subgroup tokens are associated with nodes and subgroups, respectively. Each node $u$ in $S$ is associated to two node tokens, i.e. a forward node token $t_{u}^{F}$ and a backward node token $t_{u}^{B}$. Elder cognates of $u$ store token $t_{u}^{F}$, while junior cognates of $u$ store token $t_{u}^{B}$. Similarly, each subgroup $S$ is associated to two subgroup tokens, i.e. a forward subgroup token $s t_{S}^{F}$ and a backward subgroup token $s t_{S}^{B}$. All nodes in elder kindreds of $S$ store $s t_{S}^{F}$, while all nodes in junior kindreds of $S$ store $s t_{S}^{B}$. Also, every node $u$ a priori shares a node key $K_{u}$ with the KM. Every subgroup $S$ is associated to a subgroup key $K_{S}$, which is shared between the KM and every node in the subgroup. Finally, the KM stores: i) all node tokens in the Node Token Set $(N T S)$; ii) all subgroup tokens in the Subgroup Token Set $(S T S)$; iii) all node keys in the Node Key Set $(N K S)$; and iv) all subgroup keys in the Subgroup Key Set (SKS). The KM and nodes keep tokens and keys secret.

Let us consider a node $u$ in a subgroup $S$. Node $u$ holds its node key $K_{u}$, the subgroup key $K_{S}$, and the node tokens associated to its cognate nodes. Such tokens are partitioned into two sets, i.e. Backward Node Token Set $\left(N T S_{u}^{B}\right)$ and Forward Node Token Set $\left(N T S_{u}^{F}\right)$. In particular, $N T S_{u}^{B}$ includes all backward node tokens associated to the elder cognates of $u$. Instead, $N T S_{u}^{F}$ includes all forward node tokens associated to the junior cognates of $u$. Also, node $u$ holds the subgroup tokens of all subgroups belonging to the absolute complement of $S$ in $\mathcal{S}$. Such tokens are partitioned into two sets, i.e. Backward Subgroup Token Set $\left(S T S_{S}^{B}\right)$ and Forward Subgroup Token Set $\left(S T S_{S}^{F}\right)$. In particular, $S T S_{S}^{B}$ includes all backward subgroup tokens associated to the elder kindreds of $S$, while $S T S_{S}^{F}$ includes all forward subgroup tokens associated to the junior kindreds of $S$.

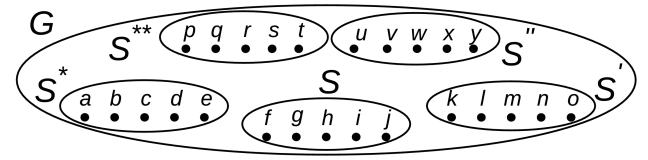

Figure 1. A group $G$ partitioned into five subgroups.

To fix ideas, we consider the example in Figure 1, where a group $G$ is partitioned into five subgroups, each one
(A)

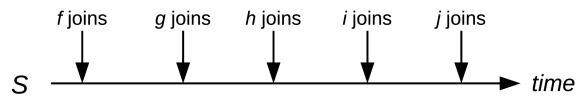

(B)

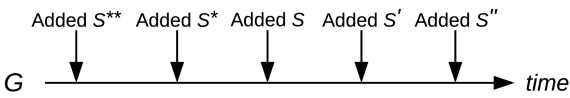

Figure 2. (A) Node join history of $S$; (B) Subgroup addition history.

TABLE 1. NODE AND SUBGROUP TOKENS.

\begin{tabular}{|c|c|c|c|c|c|}
\hline Subgroup & Node & $N T S^{B}$ & $N T S^{F}$ & $S T S^{B}$ & $S T S^{F}$ \\
\hline$S^{*}$ & $\begin{array}{l}a \\
b \\
c \\
d \\
e \\
\end{array}$ & $\begin{array}{c}- \\
t_{a}^{B} \\
t_{a}^{B}, t_{b}^{B} \\
t_{a}^{B}, t_{b}^{B}, t_{c}^{B} \\
t_{a}^{B}, t_{b}^{B}, t_{c}^{B}, t_{d}^{B}\end{array}$ & $\begin{array}{c}t_{b}^{F}, t_{c}^{F}, t_{d}^{F}, t_{e}^{F} \\
t_{c}^{F}, t_{d}^{F}, t_{e}^{F} \\
t_{d}^{F}, t_{e}^{F} \\
t_{e}^{F} \\
- \\
-\end{array}$ & $\begin{array}{l}s t_{S^{* *}}^{B} \\
s t_{S^{* *}}^{B} \\
s t_{S^{* *}}^{B} \\
s t_{S^{* *}}^{B} \\
s t_{S^{* *}}^{B}\end{array}$ & $\begin{array}{l}s t_{S}^{F}, s t_{S^{\prime}}^{F}, s t_{S^{\prime \prime}}^{F} \\
s t_{S}^{F}, s t_{S^{\prime}}^{F}, s t_{S^{\prime \prime}}^{F} \\
s t_{S}^{F}, s t_{S^{\prime}}^{F}, s t_{S^{\prime \prime}}^{F} \\
s t_{S}^{F}, s t_{S^{\prime}}^{F}, s t_{S^{\prime \prime}}^{F} \\
s t_{S}^{F}, s t_{S^{\prime}}^{F}, s t_{S^{\prime \prime}}^{F}\end{array}$ \\
\hline$S$ & $\begin{array}{l}f \\
g \\
h \\
h \\
i \\
j\end{array}$ & $\begin{array}{c}- \\
t_{f}^{B} \\
t_{f}^{B}, t_{g}^{B} \\
t_{f}^{B}, t_{g}^{B}, t_{h}^{B} \\
t_{f}^{B}, t_{g}^{B}, t_{h}^{B}, t_{i}^{B}\end{array}$ & $\begin{array}{c}t_{g}^{F}, t_{h}^{F}, t_{i}^{F}, t_{j}^{F} \\
t_{h}^{F}, t_{i}^{F}, t_{j}^{F} \\
t_{i}^{F}, t_{j}^{F} \\
t_{j}^{F} \\
- \\
-\end{array}$ & $\begin{array}{l}s t_{S^{* *}}^{B}, s t_{S^{*}}^{B} \\
s t_{S^{* *}}^{B}, s t_{S^{*}}^{B} \\
s t_{S^{* *}}^{B}, s t_{S^{*}}^{B} \\
s t_{S^{* *}}^{B}, s t_{S^{*}}^{B} \\
s t_{S^{* *}}^{B}, s t_{S^{*}}^{B}\end{array}$ & $\begin{array}{l}s t_{S^{\prime}}^{F}, s t_{S^{\prime \prime}}^{F} \\
s t_{S^{\prime}}^{F}, s t_{S^{\prime \prime}}^{F} \\
s t_{S^{\prime}}^{F} s t_{S^{\prime \prime}}^{F} \\
s t_{S^{\prime}}^{F}, s t_{S^{\prime \prime}}^{F} \\
s t_{S^{\prime}}^{F}, s t_{S^{\prime \prime}}^{F}\end{array}$ \\
\hline$S^{\prime}$ & $\begin{array}{l}k \\
l \\
m \\
n \\
o\end{array}$ & $\begin{array}{c}- \\
t_{k}^{B} \\
t_{k}^{B}, t_{l}^{B} \\
t_{k}^{B}, t_{l}^{B}, t_{m}^{B} \\
t_{k}^{B}, t_{l}^{B}, t_{m}^{B}, t_{n}^{B}\end{array}$ & $\begin{array}{c}t_{l}^{F}, t_{m}^{F}, t_{n}^{F}, t_{o}^{F} \\
t_{m}^{F}, t_{n}^{F}, t_{o}^{F} \\
t_{n}^{F}, t_{o}^{F} \\
t_{o}^{F} \\
- \\
-\end{array}$ & $\begin{array}{l}s t_{S^{* *}}^{B}, s t_{S^{*}}^{B}, s t_{S}^{B} \\
s t_{S^{* *}}^{B}, s t_{S^{*}}^{B}, s t_{S}^{B} \\
s t_{S^{* *}}^{B} s t_{S^{*}}^{B}, s t_{S}^{B} \\
s t_{S^{* *}}^{B} s t_{S^{*}}^{B}, s t_{S}^{B} \\
s t_{S^{* *}}^{B}, s S_{S^{*}}^{B}, s t_{S}^{B}\end{array}$ & $\begin{array}{l}s t_{S^{\prime \prime}}^{F} \\
s t_{S^{\prime \prime}}^{F} \\
s t_{S^{\prime \prime}}^{F} \\
s t_{S^{\prime \prime}}^{F} \\
s t_{S^{\prime \prime}}^{F}\end{array}$ \\
\hline
\end{tabular}

including five nodes. Figure 2(A) shows the node join history of subgroup $S$, while Figure 2(B) shows the subgroup addition history of $G$. Finally, Table 1 shows the node and subgroup tokens held by nodes in subgroups $S^{*}, S$ and $S^{\prime}$.

Hereafter, we adopt the following notation. By $P \rightarrow$ $u: m$ we denote a principal $P$ sending a unicast message $m$ to node $u$. By $P \nrightarrow S: m$ we denote $P$ that broadcasts a message $m$ to (sub)group $S$. We denote by $H(\cdot)$ a one-way hash function, and by $K D F(\cdot)$ a pseudo-random key derivation function that derives one cryptographic key from a secret value. By $\{x\}_{K}$, we denote the symmetric encryption of $x$ by means of key $K$. We assume that cryptographic primitives are secure, and secrets have a size that discourages an exhaustive search, thus no analytical attack against rekeying and data traffic is practically feasible. Due to space constraints, we do not cover how to assure integrity and authenticity of rekeying messages. Possible mechanisms to provide them are digital signatures and hash-chains [6].

\subsection{Rekeying upon joining}

Let us consider a node $u$ joining the group $G$. We assume that $u$ is not malicious or compromised, and has been authorized to join the group by the GMS. Due to space constraints, we consider the association of $u$ to an already existing subgroup $S$. The KM renews the group security material as follows, in order to assure backward security.

The KM randomly generates a refresh key $K_{R}$, a node key $K_{u}$, a backward node token $t_{u}^{B}$ and a master node token $t_{M}$. Then, it derives a forward node token $t_{u}^{F}=K D F\left(t_{M} \| K_{R}\right)$. After that, it determines a node ID $n i d_{u}$ associated to $u$, and computes a new group key 
$K_{G}^{+}=K D F\left(K_{G} \| K_{R}\right)$ and a new subgroup key $K_{S}^{+}=$ $K D F\left(K_{S} \| K_{R}\right)$. Finally, the KM broadcasts the following messages:

$$
\begin{aligned}
& \text { JM1 }: K M \nrightarrow S:<\operatorname{nid}_{u},\left\{t_{M}, K_{R}\right\}_{K_{S}}> \\
& \text { JM2 }: K M \nrightarrow G:<\left\{K_{R}\right\}_{K_{G}}>
\end{aligned}
$$

That is, the KM rekeys $S$ by means of JM1, and the remaining subgroups by means of JM2. Then, it installs $K_{G}^{+}$ as the current group key, and $K_{S}^{+}$as the current subgroup key of subgroup $S$. Finally, it adds $t_{u}^{B}$ and $t_{u}^{F}$ to $N T S$, and discards $t_{M}$ and $K_{R}$.

Upon receiving message $\mathrm{JM} 1$, any node $v$ in $S$ uses $K_{S}$ to retrieve $t_{M}$ and $K_{R}$. Then, it derives $t_{u}^{F}=$ $K D F\left(t_{M} \| K_{R}\right)$ and adds it to $N T S_{v}^{F}$. Also, it generates the two keys $K_{G}^{+}=\operatorname{KDF}\left(K_{G} \| K_{R}\right)$ and $K_{S}^{+}=$ $K D F\left(K_{S} \| K_{R}\right)$, and installs them as the current group key and subgroup key, respectively. Finally, it discards $t_{M}$ and $K_{R}$. Upon receiving message $\mathrm{JM} 2$, any node $v$ in $S^{\prime}, S^{\prime} \neq S$ : i) uses $K_{G}$ to retrieve $K_{R}$; ii) generates $K_{G}^{+}=K D F\left(K_{G} \| K_{R}\right)$ and installs it as the current group key; and iii) discards $K_{R}$.

Before joining the group, $u$ initializes its token sets as empty sets. Then, the $\mathrm{KM}$ provides $u$ with $K_{u}, K_{G}^{+}$ and $K_{S}^{+}$. Upon receiving them, $u$ installs $K_{u}$ as its own node key, $K_{G}^{+}$as the current group key, and $K_{S}^{+}$as the current subgroup key. Then, node $u$ receives from the KM the backward node tokens associated to its cognates in $S$, and adds them to $N T S_{u}^{B}$. Finally, the KM provides $u$ with the backward and forward subgroup tokens associated to the elder and junior kindreds of $S$. Upon receiving them, $u$ accordingly adds the subgroup tokens to $S T S_{S}^{B}$ and $S T S_{S}^{F}$. We assume that node $u$ receives the cryptographic material through a pre-existing secure channel, so assuring authentication and confidentiality. Possible implementations include a pre-shared cryptographic key or out-of-band means.

\subsection{Rekeying upon leaving}

With reference to Figure 1, let us suppose that node $h$ in subgroup $S$ leaves the group $G$. Then, all the cryptographic material held by $h$, including the group key $K_{G}$, gets compromised and must be revoked. In particular, a new group key must be distributed to all nodes in $G$ but $h$. Due to space constraints, we do not discuss the case when $h$ is the only member of subgroup $S$, which never becomes empty.

To rekey the group in a scalable way, the KM uses tokens, and bases the rekeying on the following observation. When node $h$ leaves the group, all the tokens it holds get compromised (see Table 1). However, by construction, four tokens remain secret, i.e. $t_{h}^{B}, t_{h}^{F}, s t_{S}^{F}$ and $s t_{S}^{B}$. The KM can thus rely on these tokens to efficiently rekey the group. In fact, i) all elder cognates of $h$ (nodes $f$ and $g$ ) hold $t_{h}^{F}$; ii) all junior cognates of $h$ (nodes $i$ and $j$ ) hold $t_{h}^{B}$; iii) nodes in all elder kindreds of $S$ (subgroups $S^{*}$ and $S^{* *}$ ) hold $s t_{S}^{F}$; iv) nodes in all junior kindreds of $S$ (subgroups $S^{\prime}$ and $S^{\prime \prime}$ ) hold $\left.s t_{S}^{B} ; \mathrm{v}\right)$ node $h$ does not know either $t_{h}^{F}$ or $t_{h}^{B}$; and vi) no node in $S$, including node $h$, holds $s t_{S}^{F}$ or $s t_{S}^{B}$.
Practically, the KM randomly generates a refresh key $K_{R}$, and computes the new group key $K_{G}^{+}=$ $\operatorname{KDF}\left(K_{G} \| K_{R}\right)$ and the new subgroup key $K_{S}^{+}=$ $K D F\left(K_{S} \| K_{R}\right)$. Then, it computes the four key encryption keys $K_{F}=K D F\left(t_{h}^{F}\right), K_{B}=K D F\left(t_{h}^{B}\right), K_{F}^{S}=$ $K D F\left(s t_{S}^{F}\right)$, and $K_{B}^{S}=K D F\left(s t_{S}^{B}\right)$. Finally, it broadcasts the following messages:

$$
\begin{array}{ll}
\text { LM1 } & K M \nrightarrow S:<\operatorname{nid}_{h},\left\{K_{R}\right\}_{K_{F}},\left\{K_{R}\right\}_{K_{B}}> \\
\text { LM2 } & K M \nrightarrow G:<\operatorname{sid}_{S},\left\{K_{R}\right\}_{K_{F}^{S}},\left\{K_{R}\right\}_{K_{B}^{S}}>
\end{array}
$$

That is, the KM rekeys $S$ by means of LM1, and the remaining subgroups by means of LM2. Then, it installs $K_{G}^{+}$as the current group key and $K_{S}^{+}$as the current subgroup key of subgroup $S$. Then, it updates its node token set and subgroup token set by means of $K_{R}$, as follows. First, it removes the two node tokens $t_{h}^{B}$ and $t_{h}^{F}$ associated to $h$ from its node token set NTS. Then, it updates all the node tokens in NTS associated to the remaining nodes in $S$, i.e. $\forall t_{u} \in N T S$, s.t. $u$ belongs to $S$ and $u \neq h, t_{u} \leftarrow H\left(t_{u} \| K_{R}\right)$. Also, the $\mathrm{KM}$ updates all subgroup tokens in its subgroup token set $S T S$, i.e. $\forall s t \in S T S, s t \leftarrow H\left(s t|| K_{R}\right)$. Finally, the $\mathrm{KM}$ discards $K_{R}, K_{F}, K_{B}, K_{F}^{S}$ and $K_{B}^{S}$.

Upon receiving message LM1, any node $u$ in $S, u \neq h$, computes either $K_{F}=K D F\left(t_{h}^{F}\right)$, if $n i d_{u}<n i d_{h}$, or $K_{B}=$ $K D F\left(t_{h}^{B}\right)$ otherwise. Then, it retrieves $K_{R}$, and computes $K_{G}^{+}=K D F\left(K_{G} \| K_{R}\right)$ and $K_{S}^{+}=K D F\left(K_{S} \| K_{R}\right)$. Such keys are installed as the current group key and subgroup key, respectively. Also, $u$ removes either $t_{h}^{B}$ from the node token set $N T S_{u}^{B}$ or $t_{h}^{F}$ from the node token set $N T S_{u}^{F}$. Then, $u$ updates all tokens in its token sets, i.e. $\forall T S \in$ $\left\{N T S_{u}^{B}, N T S_{u}^{F}, S T S_{S}^{B}, S T S_{S}^{F}\right\}, \forall t \in T S, t \leftarrow H\left(t \| K_{R}\right)$. Finally, $u$ discards $K_{R}$, as well as either $K_{F}$ or $K_{B}$.

Upon receiving message LM2, any node $v$ in $S^{\prime}, S^{\prime} \neq$ $S$, computes either $K_{F}^{S}=K D F\left(s t_{S}^{F}\right)$, if $\operatorname{sid}_{S^{\prime}}<\operatorname{sid}_{S}$, or $K_{B}^{S}=K D F\left(s t_{S}^{B}\right)$ otherwise. Then, it retrieves the key $K_{R}$, computes $K_{G}^{+}=K D F\left(K_{G} \| K_{R}\right)$, and installs it as the current group key. Also, $v$ updates its subgroup token sets, i.e. $\forall T S \in\left\{S T S_{S^{\prime}}^{B}, S T S_{S^{\prime}}^{F^{\prime}}\right\}, \forall t \in T S, t \leftarrow H\left(t \| K_{R}\right)$. Finally, node $v$ discards $K_{R}$, as well as either $K_{F}^{S}$ or $K_{B}^{S}$.

\subsection{Recovering from collusion attack}

In case of collusion attack, multiple compromised nodes may share their individual pieces of information to regain access to the group key. The compromised nodes can all belong to the same subgroup, or be spread among different subgroups. Hereafter, we call compromised subgroup any subgroup containing at least one compromised node. Generally, recovering from collusion might require a total member reinitialization, i.e. all non compromised nodes in the group require to be reinitialized one by one. This would greatly limit efficiency and scalability of the rekeying process.

On the contrary, GREP efficiently recovers from collusion attacks, by following two intuitive observations. First, with reference to Figure 2, let us assume that nodes $g$ and $i$, nid $d_{g}<n i d_{i}$, belonging to subgroup $S$, are colluding, i.e. 
an adversary can collect all the tokens and keys they hold. By construction, all node tokens in $S$ get compromised but two, i.e. $t_{i}^{B}$ and $t_{g}^{F}$ (see Table 1). That is, all junior cognates of $i$, e.g. node $j$, hold the former, while all elder cognates of node $g$, e.g. node $f$, hold the latter. Second, with reference to Figure 2, let us assume that $S^{*}$ and $S^{\prime}, \operatorname{sid}_{S^{*}}<\operatorname{sid}_{S^{\prime}}$, are compromised. By construction, all subgroup tokens get compromised but two, i.e. $s t_{S^{\prime}}^{B}$ and $s t_{S^{*}}^{F}$ (see Table 1). That is, all junior kindreds of $S^{\prime}$, e.g. $S^{\prime \prime}$, hold the former, while all elder kindreds of $S^{*}$, e.g. $S^{* *}$, hold the latter.

Let us denote with $G_{c}$ the set of colluding nodes to be evicted, with $\mathcal{C}$ the set of compromised subgroups, and with $\mathcal{U}=\mathcal{S} \backslash \mathcal{C}$ the set of non compromised subgroups. Due to space constraints, we assume that: i) all compromised subgroups include at least one non compromised node, i.e. no compromised subgroups become empty after the recovery has been completed; and ii) no previously evicted nodes collude with nodes in $G_{c}$. Then, the KM revokes the current group key $K_{G}$ and distributes a new one $K_{G}^{+}$as follows.

1) Initially, the KM randomly generates a refresh key $K_{R}$. Then, it computes a new group key $K_{G}^{+}=K D F\left(K_{G} \| K_{R}\right)$ and installs it as the current group key.

2) The KM rekeys the compromised subgroups as follows. For each subgroup $S \in \mathcal{C}$, it determines: i) the subset $S_{c}$ of compromised nodes in $S$; and ii) $u_{y}^{S}$ and $u_{e}^{S}$, i.e. the youngest and the eldest node in $S_{c}$, respectively. Practically, the set $S_{c}$ can be represented as a list of node IDs. According to the first observation, node tokens $t_{u_{e}^{S}}^{F}$ and $t_{u_{y}^{S}}^{B}$ are not compromised. Also, all elder cognates of $u_{e}^{S}$ hold token $t_{u_{e}^{S}}^{F}$, while all junior cognates of $u_{y}^{S}$ hold token $t_{u_{y}^{S}}^{B}$. Hence, these tokens can be used to rekey these nodes as follows. The $\mathrm{KM}$ generates the key encryption keys $K_{F}=K D F\left(t_{u_{e}^{S}}^{F}\right)$ and $K_{B}=K D F\left(t_{u_{y}^{S}}^{B}\right)$, and broadcasts the message

$$
\text { RM1 } K M \nrightarrow S:<S_{c},\left\{K_{R}\right\}_{K_{F}},\left\{K_{R}\right\}_{K_{B}}>
$$

Every non compromised node $u$ which is elder cognate of $u_{y}^{S}$ and junior cognate of $u_{e}^{S}$ holds only compromised tokens. Thus, it must be rekeyed in a one-to-one fashion, by means of its user key $K_{u}$. Then, $\forall u$ in $S \backslash S_{c}$, such that $\operatorname{nid}_{u_{e}^{S}}<n i d_{u}<\operatorname{nid}_{u_{y}^{S}}$, the KM sends the message

$$
\mathrm{RM} 2 K M \rightarrow u:<\left\{S_{c}, K_{R}\right\}_{K_{u}}>
$$

3) The KM rekeys the non compromised subgroups in $\mathcal{U}$, as follows. It determines the subgroups $S_{y}^{\mathcal{C}}$ and $S_{e}^{\mathcal{C}}$, i.e. the youngest and the eldest subgroup in $\mathcal{C}$, respectively. By construction, subgroup tokens $s t_{S_{e}^{\mathcal{C}}}^{F}$ and $s t_{S_{y}^{\mathcal{C}}}^{B}$ are not compromised. Also, all elder kindreds of $S_{e}^{\mathcal{C}}$ hold token $s t_{S_{e}^{\mathcal{C}}}^{F}$, while all junior kindreds of $S_{y}^{\mathcal{C}}$ hold token $s t_{S_{y}^{\mathcal{C}}}^{B}$. These tokens are used to rekey these subgroups, i.e. the $\mathrm{KM}$ generates the key encryption keys $K_{F}^{S}=K D F\left(s t_{S_{e}^{C}}^{F}\right)$ and $K_{B}^{S}=K D F\left(s t_{S_{y}^{\mathcal{C}}}^{B}\right)$, and broadcasts the message

RM3 $K M \nrightarrow G:<\operatorname{sid}_{S_{e}^{c}}, \operatorname{sid}_{S_{y}^{c}},\left\{K_{R}\right\}_{K_{F}^{S}},\left\{K_{R}\right\}_{K_{B}^{S}}>$
Every other non compromised subgroup $S$ which is elder kindred of $S_{y}^{\mathcal{C}}$ or junior kindred of $S_{e}^{\mathcal{C}}$ holds only compromised subgroup tokens. Thus, it must be rekeyed by means of its subgroup key $K_{S}$. Then, $\forall S \in \mathcal{U}$, such that $\operatorname{sid}_{S_{e}^{\mathcal{C}}}<\operatorname{sid}_{S}<\operatorname{sid}_{S_{y}^{\mathcal{C}}}$, the KM broadcasts the message

$$
\text { RM4 } K M \nrightarrow S:<\operatorname{sid}_{S_{e}^{\mathcal{C}}}, \operatorname{sid}_{S_{y}^{\mathcal{C}}},\left\{K_{R}\right\}_{K_{S}}>
$$

4) The KM updates its token sets. First, it removes the node tokens $t_{v}^{B}$ and $t_{v}^{F}$ associated to each compromised node $v \in G_{c}$ from NTS. Then, it updates the remaining node tokens in NTS, i.e. $\forall t_{u} \in N T S$, s.t. $u$ belongs to $S \in \mathcal{C}$ and $u \notin G_{c}, t_{u} \leftarrow H\left(t_{u} \| K_{R}\right)$. Also, the KM updates all subgroup tokens in $S T S$, i.e. $\forall s t \in S T S, s t \leftarrow H\left(s t|| K_{R}\right)$. Then, for each $S \in \mathcal{C}$, the $\mathrm{KM}$ computes $K_{S}^{+}=\operatorname{KDF}\left(K_{S} \| K_{R}\right)$ and installs it as the current subgroup key of $S$. Finally, the KM discards $K_{R}$.

On their side, nodes perform the following steps.

- Every non compromised node $u$ in a compromised subgroup $S$, i.e. $\forall S \in \mathcal{C}, \forall u$ in $S \backslash S_{c}$, retrieves $K_{R}$ from RM1 if $\left(n i d_{u}<n i d_{u_{e}}\right.$ or $\left.n i d_{u}>n_{i} d_{u_{y}^{S}}\right)$, or from RM2 otherwise. Then, $u$ computes $K_{S}^{+}=\operatorname{KDF}\left(K_{S} \| K_{R}\right)$ and installs it as the current subgroup key. Also, $u$ removes the node tokens associated to all compromised nodes in $S$, i.e. $\forall v$ in $S_{c}, N T S_{u}^{B} \leftarrow N T S_{u}^{B} \backslash\left\{t_{v}^{B}\right\}$ and $N T S_{u}^{F} \leftarrow N T S_{u}^{F} \backslash\left\{t_{v}^{F}\right\}$. Finally, node $u$ updates all remaining node tokens, i.e. $\forall T S \in\left\{N T S_{u}^{B}, N T S_{u}^{F}\right\}, \forall t \in T S, t \leftarrow H\left(t \| K_{R}\right)$.

- Every (non compromised) node $u$ in a non compromised subgroup $S$, i.e. $\forall S \in \mathcal{U}, \forall u$ in $S$, retrieves $K_{R}$ from RM4 if $\operatorname{sid}_{S_{e}^{c}}<\operatorname{sid}_{S}<\operatorname{sid}_{S_{y}^{c}}$, or from RM3 otherwise.

- Every rekeyed node $u$ computes $K_{G}^{+}=K D F\left(K_{G} \| K_{R}\right)$ and installs it as the current group key. Also, $u$ updates its subgroup token sets, i.e. $\forall S T \in\left\{S T S_{u}^{B}, S T S_{u}^{F}\right\}, \forall t \in$ $S T, t \leftarrow H\left(t|| K_{R}\right)$. Finally, $u$ discards $K_{R}$.

\section{Security analysis}

Backward security. Let us consider a node $u$ that joins the group $G$ as a member of subgroup $S$. The issue is to prove that $u$ cannot access the current group key $K_{G}$ and subgroup key $K_{S}$, or any previous incarnation of them. In the join rekeying procedure, the KM achieves this goal by first rekeying $S$ and $G$, and then initializing $u$. Actually, before $u$ can join the group $G$, the KM efficiently rekeys all the current members of $G$ (but $u$ ), by means of messages JM1 and JM2, so causing the installation of the new keys $K_{S}^{+}$and $K_{G}^{+}$. Confidentiality of JM1 and JM2 is protected by means of $K_{S}$ and $K_{G}$, respectively. Thus, only members of $S$ and $G$ (but $u$ ) can decrypt them, retrieve the secret material therein contained, and generate the new keys $K_{S}^{+}$and $K_{G}^{+}$. Only once this process has been successfully completed, $u$ receives the new keys $K_{S}^{+}$and $K_{G}^{+}$. Therefore, $u$ never gets 
knowledge of the security material used before its join, and therefore is not able to access old group communication.

Forward security. There are two cases to consider, i.e. node leaving and recovery from collusion attack. Let us first consider the former case of a leaving node $h$ in a subgroup $S$. The issue is to prove that the leaving node $h$ cannot take part to the rekeying process, and hence cannot get access to the new subgroup key $K_{S}^{+}$and group key $K_{G}^{+}$, or any future incarnation of them. In the leave rekeying procedure, the KM achieves this goal in two steps. In the first step, the KM rekeys subgroup $S$ by means of message LM1, which is encrypted by means of (keys deriving from) node tokens $t_{h}^{F}$ and $t_{h}^{B}$. Then, in the second step, the KM rekeys the remaining subgroups by means of message LM2, which is encrypted by means of (keys deriving from) subgroup tokens $s t_{S}^{F}$ and $s t_{S}^{B}$. By construction, $h$ does not hold such tokens. As a consequence, it cannot access messages LM1 and LM2, and thus is excluded from the rekeying process.

Let us now consider the case of recovery from collusion attack, and the consequent eviction of multiple colluding nodes. The issue here is to prove that the leaving nodes, even when colluding, cannot take part to the rekeying process, and hence cannot get access to the new subgroup key $K_{S}^{+}$ and group key $K_{G}^{+}$, or any future incarnation of them. In the collusion recovery procedure, the $\mathrm{KM}$ achieves this goal in two steps. In the first step, the KM rekeys every compromised subgroup $S \in \mathcal{C}$ by sending a single message RM1, and one message RM2 for each node $u$ in $S \backslash S_{c}$ such that nid $_{u_{e}^{S}}<n i d_{u}<n i d_{u_{y}^{S}}$. Messages RM1 and RM2 are encrypted by means of (keys deriving from) node tokens $t_{u_{e}^{S}}^{F}$ and $t_{u_{y}^{S}}^{B}$, and node keys $K_{u}$, respectively. By construction, colluding nodes in $S$ do not hold such tokens and keys, and thus cannot access messages RM1 and RM2. Then, in the second step, the KM rekeys the remaining non compromised subgroups, by sending a single message RM3, and one message RM4 for each subgroup $S \in \mathcal{U}$ such that $\operatorname{sid}_{S_{e}^{\mathcal{C}}}<\operatorname{sid}_{S}<\operatorname{sid}_{S_{y}^{c}}$. Messages RM3 and RM4 are encrypted by means of (keys deriving from) subgroup tokens $s t_{S_{e}^{\mathcal{C}}}^{F}$ and $s t_{S_{u}^{\mathcal{C}}}^{B}$, and subgroup keys $K_{S}$, respectively. By construction, colluding nodes in $G$ do not hold such tokens and keys, and thus cannot access messages RM3 and RM4. Hence, leaving nodes can never access rekeying messages. Since we assume that cryptanalytical and key exhaustive attacks are practically infeasible, leaving nodes cannot derive $K_{G}^{+}$, nor any future group key, and thus are not able to access future group communication.

\section{Performance evaluation}

We analytically evaluate GREP in terms of storage, communication, and computing overhead of rekeying upon node joining, node leaving and recovering from collusion. In particular, we evaluate storage and communication overhead as the number of information items that protocol actors store and transmit/receive, respectively, and the computing overhead as the number of performed cryptographic operations, i.e. encryptions, decryptions and hash function executions.
We consider a group $G$ composed of $p$ subgroups with $m$ nodes each, i.e. $n=p \cdot m$. GREP well supports heterogeneous subgrouping, but a homogeneous one allows us to evaluate performance with no significant lack of generality. We assume that node IDs and subgroup IDs have the same size of tokens and keys, and that the key generator, $H(\cdot)$ and $K D F(\cdot)$ result in a comparable computing overhead.

In order to give a concrete insight of the high scalability and practical sustainability of GREP, we discuss the overheads with reference to a WSN application that features a group composed of $n=1024$ TmoteSky sensor nodes, interconnected through an IEEE 802.15.4 wireless network and equipped with the Skipjack cipher [8]. Although GREP is general and not especially designed for WSNs, they constitute a challenging case study, as they are composed of a large set of interconnected resource scarce devices.

\subsection{Storage overhead}

The KM stores the group key, $p$ subgroup keys, $n$ node keys, $(2 \cdot n-2 \cdot p)$ node tokens, and $(2 \cdot p-2)$ subgroup tokens. The resulting storage overhead for the $\mathrm{KM}$ is $O_{s, k m}=(3$. $n+p-1)$, i.e. it grows linearly with $n$. This is not a problem in practice, since the KM has plentiful of resources.

Instead, it is vital that the storage overhead is affordable at the node side. Each node $u$ in a subgroup $S$ stores: i) its node key $K_{u}$; ii) the group key $K_{G}$; iii) the subgroup key $K_{S}$; iv) $(m-1)$ node tokens associated to its cognate nodes; and v) $(p-1)$ subgroup tokens associated to all subgroups but $S$. The resulting storage overhead for a node is $O_{s, u}=(p+m+1)$. If we consider $p \gg 1$ or $m \gg 1$, then $O_{s, u} \simeq p+m$. Hence, if nodes are uniformly distributed in $p$ subgroups of $m$ members each $(p=m=\sqrt{n})$, the minimum storage overhead is $O_{s, u}^{(\min )}=(2 \cdot \sqrt{n})$, i.e. it grows as $\mathcal{O}(\sqrt{n})$.

In the WSN application, the minimum storage overhead is $O_{s, u}^{(\min )}=64$. If we consider 80 bit tokens and Skipjack keys, then the storage overhead is 640 bytes. As TmoteSky nodes feature 48 Kbytes of memory, the storage overhead is equal to $1.30 \%$ of the total memory. It follows that GREP is practically affordable even in constrained sensor nodes.

\subsection{Overhead of node joining}

Node $u$ 's joining of group $G$ as a member of subgroup $S$ requires to: i) broadcast message JM1 carrying $n i d_{u}$, the master node token $t_{M}$, and the refresh key $K_{R}$; and ii) broadcast message JM2 carrying the refresh key $K_{R}$. Thus, the communication overhead amounts to four, i.e. $O_{c}^{(j)}=4$.

The worst case for the computing overhead regards the current nodes in $S$. Each of them performs: i) one decryption to retrieve $t_{M}$ and $K_{R}$ from message JM1; and ii) three hash function executions to compute $t_{u}^{F}, K_{G}^{+}$and $K_{S}^{+}$. Instead, the KM: i) computes four keys (i.e. $K_{G}^{+}, K_{S}^{+}, K_{R}$ and $K_{u}$ ), the master node token $t_{M}$, and two node tokens (i.e. $t_{u}^{B}$ and $\left.t_{u}^{F}\right)$; and ii) encrypt messages JM1 and JM2. Thus, the KM performs 2 encryptions and 7 hash function executions. 


\subsection{Overhead of node leaving}

Let us consider a node $h$ in subgroup $S$ that leaves the group $G$. Message LM1 introduces a communication overhead equal to three, as it conveys $n i d_{h}$ and two copies of $K_{R}$. Message LM2 introduces a communication overhead equal to three, as it conveys $\operatorname{sid}_{S}$ and two copies of $K_{R}$. The total communication overhead is equal to $O_{c}^{(l)}=6$. Thus, GREP efficiently rekeys the group, displaying a small and constant communication overhead which is independent of the group size, i.e. $\mathcal{O}(1)$. This makes GREP highly efficient and scalable with the number of nodes in the group.

The worst case for the computing overhead regards a node $u \neq h$ in $S$. Such node: i) computes either $K_{F}$ or $K_{B}$; ii) decrypts either $\left\{K_{R}\right\}_{K_{F}}$ or $\left\{K_{R}\right\}_{K_{B}}$ to retrieve $K_{R}$ from message LM1; iii) computes $K_{G}^{+}$and $K_{S}^{+}$; and iv) updates its node token sets and subgroup token sets by executing $(m-2)$ and $(p-1)$ hash functions, respectively. Thus, a node performs at most one decryption and $(p+m)$ hash function executions. Instead, the KM: i) computes the keys $K_{G}^{+}, K_{S}^{+}, K_{R}, K_{F}, K_{B}, K_{F}^{S}$ and $K_{B}^{S}$; ii) encrypts two copies of $K_{R}$ in message LM1 and two copies of $K_{R}$ in message LM2; and iii) updates its node token set NTS and subgroup token set $S T S$ by executing $(2 \cdot m-4)$ and $(2$. $p-2$ ) hash functions, respectively. Thus, the KM performs 4 encryptions and $(2 \cdot p+2 \cdot m+1)$ hash function executions.

The computing overheads on the KM and nodes grow both as $\mathcal{O}(\sqrt{n})$. In particular, in the WSN application, a node performs at most 1 decryption and 64 hash function executions, which is practically affordable for sensor platforms.

\subsection{Overhead of collusion recovery}

Let us consider a collusion attack with $C$ compromised subgroups and $(p-C)$ non compromised subgroups. Also, let us assume that each compromised subgroup contains $c$ compromised nodes. In general, the communication overhead of the collusion recovery depends on the specific compromised nodes and subgroups, i.e. the relation between their node ID and their cognates' and between their subgroup ID and their kindreds', respectively. In the following, we discuss the collusion recovery in the worst case condition.

We have the worst case condition when the following two events occur at the same time: 1) non compromised nodes in every compromised subgroup $S \in \mathcal{C}$ are rekeyed by means of one unicast message RM2 each, i.e. each of the $C$ compromised subgroups requires a total subgroup recovery; and 2) the $(p-C)$ non compromised subgroups $S \in \mathcal{U}$ are rekeyed by means of one broadcast message RM4 each, i.e. group $G$ requires a total group recovery.

In the worst case, $(m-c)$ unicast messages RM2 are sent within each of the $C$ compromised subgroups. The resulting communication overhead is equal to $C \cdot(m-c) \cdot(c+1)$. Also, one broadcast message RM4 is sent to each of the $(p-C)$ non compromised subgroups. The resulting communication
TABLE 2. COMMUNICATION OVERHEAD (KB).

\begin{tabular}{|c|c|c|c|c|c|}
\hline \multirow{2}{*}{$\begin{array}{c}\text { Compromised } \\
\text { subgroups }\end{array}$} & \multicolumn{5}{|c|}{ Compromised nodes per subgroup } \\
\cline { 2 - 6 } & $\mathbf{c = \mathbf { 2 }}$ & $\mathbf{c}=\mathbf{4}$ & $\mathbf{c}=\mathbf{6}$ & $\mathbf{c}=\mathbf{8}$ & $\mathbf{c}=\mathbf{1 0}$ \\
\hline $\mathbf{C}=\mathbf{1}$ & 1.88 & 2.50 & 3.13 & 3.75 & 4.38 \\
\hline $\mathbf{C}=\mathbf{1 0}$ & 10.31 & 16.56 & 22.81 & 29.06 & 35.31 \\
\hline
\end{tabular}

overhead is equal to $3 \cdot(p-C)$. Thus, the total communication overhead is $O_{c}^{(r)}=C \cdot(m-c) \cdot(c+1)+3 \cdot(p-C)$. If we reasonably assume that $i)$ each subgroup includes a non negligible number of members, i.e. $m \gg 1$; ii) only a few nodes per subgroup are captured, i.e. $m \gg c$; iii) the group $G$ includes a non negligible number of subgroups, i.e. $p \gg 1$; iv) only a few subgroups are compromised, i.e. $p \gg C$; and v) $p=m=\sqrt{n}$, for storage optimisation, then the communication overhead can be approximated as $O_{c}^{(r)} \simeq \sqrt{n} \cdot(C \cdot(c+1)+3)$. Thus, in the worst case, the communication overhead smoothly grows as $\mathcal{O}(\sqrt{n})$, and gradually increases with the severity of the attack scenario.

Table 2 shows the communication overhead in the WSN application. Even if 10 nodes in 10 different subgroups collude, i.e. $C=c=10$ and 100 nodes collude, then $O_{c}^{(r)}=35.31 \mathrm{~KB}$. In IEEE 802.15.4, unsecured frames have a payload with maximum size 102 bytes, and an implementation displays an effective data rate (excluding headers, CRCs, and control packets) of about $8.4 \mathrm{Kbps}$ (out of $250 \mathrm{Kbps}$ ). Thus, even if $C=c=10$, i.e. 100 nodes collude, the communication overhead requires 355 frames and results in $33.63 \mathrm{~s}$ (per hop). Hence, also in the worst case, the communication overhead is sustainable in a WSN environment. Note that IEEE 802.15.4 can display better performance. That is, Latré et al. showed that a throughput of about $140 \mathrm{Kbps}$ can be achieved, even if acknowlegment frames are trasmitted [1]. In that case, when $C=c=10$, the communication overhead results in $2.02 \mathrm{~s}$ (per hop).

In the worst case, the KM performs $C \cdot(m-c)+p-C$ encryptions and $C \cdot(2 \cdot(m-c)-1)+2 \cdot p-1$ hash function executions. While this is generally not a problem on the KM, the computing overhead must be practically sustainable on the node side. A non compromised node processes only one rekeying message, i.e. either RM2 or RM4, and retrieves the refresh key $K_{R}$ by performing one decryption. The highest computing overhead is experienced by a node in a compromised subgroup $S$, as it computes: i) the new group key $K_{G}^{+}$; ii) the new subgroup key $K_{S}^{+}$; iii) $(m-c-1)$ new node tokens associated to its non compromised cognate nodes; and iv) $(p-1)$ new subgroup tokens associated to the kindred subgroups of $S$. Hence, a node performs at most one decryption and $(p+m-c)$ hash function executions.

In the WSN application, the Skipjack key $K_{R}$ is only 80 bits in size. If hash functions are implemented through the same cipher Skipjack used for encryptions, then hash function executions require to process at most 640 bytes. On TmoteSky nodes, a software version of Skipjack takes $77 \mu$ s per encrypted/decrypted byte [6]. Thus, decrypting $K_{R}$ takes $0.77 \mathrm{~ms}$, and performing all the hash function 
executions takes at most $49.28 \mathrm{~ms}$, so making collusion recovery affordable from the computing standpoint.

Now, we discuss the probability $P_{w c}(G)$ of a worst case recovery to occur. Due to space constraints, we only present the results for the WSN application, and give intuitions of the attack configurations that lead to a worst case recovery.

Let us refer to Figure 2. Subgroup $S$ requires a total subgroup recovery if any of these pairs of nodes is compromised: i) $\{f, j\}$; ii) $\{f, i\}$; or iii) $\{g, j\}$, i.e. either $f=u_{e}^{S}$ or $g=u_{e}^{S}$, and either $i=u_{y}^{S}$ or $j=u_{y}^{S}$. Thus, one can not exploit node tokens in $S$ to rekey multiple nodes through a single broadcast message RM1, and each non compromised node in $S$ is rekeyed through one unicast message RM1 or RM2. Also, the more compromised nodes in a given subgroup, the more it requires a total subgroup recovery.

The group $G$ requires a total group recovery if any of these pairs of subgroups is compromised: i) $\left\{S^{* *}, S^{\prime \prime}\right\}$; ii) $\left\{S^{* *}, S^{\prime}\right\}$; or iii) $\left\{S^{*}, S^{\prime \prime}\right\}$, i.e. either $S^{* *}=S_{e}^{\mathcal{C}}$ or $S^{*}=S_{e}^{\mathcal{C}}$, and either $S^{\prime}=S_{y}^{\mathcal{C}}$ or $S^{\prime \prime}=S_{y}^{\mathcal{C}}$. Thus, one can not exploit subgroup tokens to rekey multiple subgroups through a single broadcast message RM3, and each non compromised subgroups is rekeyed through one broadcast message RM3 or RM4. Also, the more compromised subgroups, the more likely the group $G$ requires a total group recovery.

TABle 3. Probability $P_{w c}(G)$ of worst Case Recovery.

\begin{tabular}{|c|c|c|c|c|c|}
\hline \multicolumn{2}{|c|}{$\mathbf{C = 3}$} & \multicolumn{2}{c|}{$\mathbf{C}=\mathbf{5}$} & \multicolumn{2}{c|}{$\mathbf{C = 1 0}$} \\
\hline $\mathbf{c = 2}$ & $\mathbf{c}=\mathbf{1 0}$ & $\mathbf{c}=\mathbf{2}$ & $\mathbf{c}=\mathbf{1 0}$ & $\mathbf{c}=\mathbf{2}$ & $\mathbf{c}=\mathbf{1 0}$ \\
\hline $0.39 \cdot 10^{-8}$ & $0.1 \cdot 10^{-3}$ & $0.45 \cdot 10^{-12}$ & $0.31 \cdot 10^{-4}$ & $<1 \cdot 10^{-20}$ & $0.7 \cdot 10^{-7}$ \\
\hline
\end{tabular}

Table 3 shows the probability $P_{w c}(G)$ that a worst case recovery occurs. Given a number $C$ of compromised subgroups, the probability of a worst case recovery increases with $c$. This is consistent with the presence of more compromised nodes per compromised subgroup. However, given $c$ compromised nodes per compromised subgroup, the probability $P_{w c}(G)$ decreases with $C$. Intuitively, the more subgroups are compromised, the less it is likely that i) each of them requires a total subgroup recovery; and ii) all other subgroups must be separately rekeyed. If 10 nodes collude in 10 different subgroups, i.e. $C=c=10$ and 100 nodes out of 1024 collude, we have $P_{w c}(G)=0.7 \times 10^{-7}$.

Thus, a worst case recovery is an extremely unlikely event, even when a non negligible number of nodes and subgroups is compromised. Besides, a total member reinitialization is necessary only when all subgroups are compromised, i.e. $C=p$, and each of them requires a total subgroup recovery. This, together with the limited overheads displayed even in worst case conditions, makes GREP extremely efficient when recovering from collusion attacks.

\section{Conclusion}

We have presented GREP, a novel group rekeying protocol that efficiently rekeys a group with a number of messages which is small, constant and independent of the group size. In case of collusion attack, GREP recovers the group by exploiting the history of joining events. This avoids a total member reinitialization and results in an overhead which smoothly grows with the group size, and gradually increases with the attack severity. We have provided an analytical performance evaluation and shown that GREP is deployable on large-scale networks of constrained devices.

\section{Acknowledgments}

This project has received funding from the European Union's Seventh Framework Programme for research, technological development and demonstration under grant agreement no. 607109. This work was also supported by the EIT DIGITAL HII project ACTIVE; "Progetti di Ricerca di Ateneo - PRA 2016" of the University of Pisa; and the PRIN project TENACE (20103P34XC) funded by the Italian Ministry of Education, University and Research.

\section{References}

[1] B. Latré, P. De Mil, I. Moerman, N. Van Dierdonck, B. Dhoedt and P. Demeester, "Maximum Throughput and Minimum Delay in IEEE 802.15.4," in The First international conference on Mobile Ad-hoc and Sensor Networks, Wuhan, China, vol. 3794. Springer, 2005, pp. 866-876.

[2] C. K. Wong, M. Gouda and S. S. Lam, "Secure group communications using key graphs," IEEE/ACM Trans. on Networking, vol. 8, no. 1, pp. 16-30, 2000.

[3] D. Wallner, E. Harder and R. Agee, Key Management for Multicast: Issues and Architectures, IETF, 1999.

[4] E. Cole, Network Security Bible, 2nd Edition. Wiley, 2009.

[5] F. Bao, I. Chen, M. Chang and J. Cho, "Hierarchical Trust Management for Wireless Sensor Networks and its Applications to TrustBased Routing and Intrusion Detection," IEEE Trans. on Network and Service Management, vol. 9, no. 2, pp. 1-15, 2012.

[6] G. Dini and I. M. Savino, "LARK: A Lightweight Authenticated ReKeying Scheme for Clustered Wireless Sensor Networks," ACM Trans. on Embedded Computing Systems, vol. 10, no. 4, pp. 41:141:35, 2011.

[7] G. Dini and M. Tiloca, "HISS: A HIghly Scalable Scheme for Group Rekeying," The Computer Journal, vol. 56, no. 4, pp. 508-525, 2013.

[8] J. Doumen, Y. W. Law and P. H. Hartel, "Survey and benchmark of block ciphers for wireless sensor networks," ACM Trans. on Sensor Networks, vol. 2, no. 1, pp. 65-93, 2006.

[9] K. Birman, Guide to Reliable Distributed Systems. Building HighAssurance Applications and Cloud-Hosted Services. Springer, 2012.

[10] P. Liu, W.-C. Lee, Q. Gu and C.-H. Chu, "KTR: An Efficient Key Management Scheme for Secure Data Access Control in Wireless Broadcast Services," IEEE Trans. on Dependable and Secure Computing, vol. 6, no. 3, pp. 188-201, 2009.

[11] S. Rafaeli and D. Hutchison, "A Survey of Key Management for Secure Group Communication," ACM Computing Surveys, vol. 35, no. 3, pp. 309-329, 2003.

[12] S. Setia, S. Zhu and S. Jajodia, "LEAP+: Efficient security mechanisms for large-scale distributed sensor networks," ACM Trans. on Sensor Networks, vol. 2, no. 4, pp. 500-528, 2006.

[13] Y. Wang, X. Wang, B. Xie, D. Wang, and D. P. Agrawal, "Intrusion Detection in Homogeneous and Heterogeneous Wireless Sensor Networks," IEEE Transactions on Mobile Computing, vol. 7, no. 6, pp. 698-711, June 2008. 